\title{
Prognostic Impact of Prior Androgen Receptor Axis-targeting Agents in Cabazitaxel Chemotherapy After Docetaxel
}

\author{
MASAKI SHIOTA $^{1}$, MOTONOBU NAKAMURA ${ }^{2}$, AKIRA YOKOMIZO ${ }^{3}$, TOSHIHISA TOMODA ${ }^{4}$, \\ NAOTAKA SAKAMOTO ${ }^{5}$, NARIHITO SEKI $^{6}$, SHUJI HASEGAWA ${ }^{7}$, \\ TAKAKAZU YUNOKI ${ }^{8}$, MASAHIKO HARANO $^{9}$, KENTARO KUROIWA $^{10}$ and MASATOSHI ETO ${ }^{1}$ \\ ${ }^{1}$ Department of Urology, Graduate School of Medical Sciences, Kyushu University, Fukuoka, Japan; \\ ${ }^{2}$ Department of Urology, National Hospital Organization Kyushu Cancer Center, Fukuoka, Japan; \\ ${ }^{3}$ Division of Urology, Harasanshin Hospital, Fukuoka, Japan; \\ ${ }^{4}$ Department of Urology, Oita Prefectural Hospital, Oita, Japan; \\ ${ }^{5}$ Department of Urology, National Hospital Organization Kyushu Medical Center, Fukuoka, Japan; \\ ${ }^{6}$ Department of Urology, Kyushu Central Hospital, Fukuoka, Japan; \\ ${ }^{7}$ Department of Urology, Kitakyushu Municipal Medical Center, Kitakyushu, Japan; \\ ${ }^{8}$ Department of Urology, Japanese Red Cross Fukuoka Hospital, Fukuoka, Japan; \\ ${ }^{9}$ Department of Urology, JCHO Kyushu Hospital, Kitakyushu, Japan; \\ ${ }^{10}$ Department of Urology, Miyazaki Prefectural Miyazaki Hospital, Miyazaki, Japan
}

\begin{abstract}
Background/Aim: The novel taxane cabazitaxel has been shown to exert excellent anticancer effects after androgen receptor axis-targeting (ARAT) agents in clinical data, but not in in vitro data. We investigated the clinical outcome of cabazitaxel chemotherapy after docetaxel according to use of ARAT agents. Patients and Methods: Prostate specific antigen (PSA) response, progression-free survival, and overall survival were compared between cases with and without prior use of ARAT agents in 74 Japanese patients with metastatic castration-resistant prostate cancer treated with cabazitaxel chemotherapy. Results: Background characteristics were comparable between patients with and without prior use of ARAT agents. PSA response, progression-free survival, and overall survival in cabazitaxel chemotherapy were comparable between patients with and without prior use of ARAT agents. Conclusion: No detrimental effects of prior ARAT agents on clinical outcome were observed for cabazitaxel chemotherapy in the postdocetaxel setting, suggesting that cabazitaxel can be expected to remain active even after ARAT agent therapy.
\end{abstract}

Correspondence to: Masaki Shiota, MD, Ph.D., Department of Urology, Graduate School of Medical Sciences, Kyushu University, 3-1-1 Maidashi, Higashi-ku, Fukuoka 812-8582, Japan. Tel: +81 926425603, Fax: +81 926425618, e-mail: shiota@uro.med.kyushuu.ac.jp

Key Words: Abiraterone, androgen receptor, cabazitaxel, docetaxel, enzalutamide, prostate cancer.
Currently, docetaxel chemotherapy as well as androgen receptor (AR) axis-targeting (ARAT) agents such as abiraterone, enzalutamide, and apalutamide laid on androgen-deprivation therapy (ADT) are standard therapeutic strategies for metastatic hormone-sensitive prostate cancer and castration-resistant prostate cancer (CRPC) (1). Previously, it has been reported that the anticancer outcome of docetaxel chemotherapy after use of ARAT agents was inferior to that without prior use of ARAT agents $(2,3)$. However, cabazitaxel has been shown to differ from docetaxel and produce comparable anticancer outcomes with and without prior use of ARAT agents, which has been supported by in vivo experimental data (4), but not in vitro data $(3,5)$. Thus, there is a discrepancy in the anticancer effects of cabazitaxel between clinical settings and experimental models. Accordingly, we investigated the effects of prior ARAT agents on clinical outcome of cabazitaxel chemotherapy.

\section{Patients and Methods}

Patients. We enrolled patients with CRPC treated with cabazitaxel chemotherapy between 2014 and 2017 at the following institutions: Kyushu University Hospital (Fukuoka), National Hospital Organization Kyushu Cancer Center (Fukuoka), Harasanshin Hospital (Fukuoka), Oita Prefectural Hospital (Oita), National Hospital Organization Kyushu Medical Center (Fukuoka), Kyushu Central Hospital (Fukuoka), Kitakyushu Municipal Medical Center (Kitakyushu), Japanese Red Cross Fukuoka Hospital (Fukuoka), JCHO Kyushu Hospital (Kitakyushu), and Miyazaki Prefectural Miyazaki Hospital (Miyazaki). This study was approved by the 
Table I. Patient characteristics according to prior use of ARAT agents.

\begin{tabular}{|c|c|c|c|}
\hline \multirow[b]{2}{*}{ Variable } & \multicolumn{2}{|c|}{ Prior use of ARAT agents } & \multirow[b]{2}{*}{$p$-Value } \\
\hline & Absence $(n=12)$ & Presence $(n=62)$ & \\
\hline Median age at docetaxel, years (IQR) & $73(67-79)$ & $72(67-76)$ & 0.81 \\
\hline \multicolumn{4}{|l|}{ Performance status, $\mathrm{n}(\%)$} \\
\hline 0 & $8(66.7 \%)$ & $35(64.8 \%)$ & \\
\hline$\geq 1$ & $4(33.3 \%)$ & $19(35.2 \%)$ & 0.90 \\
\hline NA & 0 & 8 & \\
\hline \multicolumn{4}{|l|}{ Pain, n (\%) } \\
\hline Absence & $6(50.0 \%)$ & $31(50.0 \%)$ & \\
\hline Presence & $6(50.0 \%)$ & $31(50.0 \%)$ & 1.00 \\
\hline \multicolumn{4}{|l|}{ Biopsy Gleason score, $\mathrm{n}(\%)$} \\
\hline$\leq 8$ & $3(25.0 \%)$ & $21(35.6 \%)$ & \\
\hline$>8$ & $9(75.0 \%)$ & $38(64.4 \%)$ & 0.47 \\
\hline NA & 0 & 3 & \\
\hline Median time to CRPC, years (IQR) & $1.8(0.4-3.3)$ & $1.3(0.7-2.4)$ & 0.72 \\
\hline Median cycle of prior docetaxel (IQR) & $8(4-15)$ & $7(5-12)$ & 0.92 \\
\hline Median PSA at cabazitaxel, ng/ml (IQR) & $37.5(4.7-118.0)$ & $83.5(24.0-287.3)$ & 0.096 \\
\hline Median hemoglobin at docetaxel, g/dl (IQR) & $12.1(11.2-12.9)$ & $12.2(10.7-13.0)$ & 0.75 \\
\hline Median ALP at cabazitaxel, IU/l (IQR) & $272(181-473)$ & $284(202-521)$ & 0.78 \\
\hline Median LDH at cabazitaxel, IU/1 (IQR) & $313(185-429)$ & $245(193-330)$ & 0.45 \\
\hline \multicolumn{4}{|l|}{ Metastatic sites at docetaxel, $\mathrm{n}(\%)$} \\
\hline Lymph node & $8(66.7 \%)$ & $35(56.5 \%)$ & 0.51 \\
\hline Bone & $10(83.3 \%)$ & $56(90.3 \%)$ & 0.50 \\
\hline Visceral & $2(16.7 \%)$ & $18(29.0 \%)$ & 0.36 \\
\hline
\end{tabular}

ARAT: Androgen receptor-axis targeting; NA: not available; CRPC: castration-resistant prostate cancer; PSA: prostate-specific antigen; ALP: alkaline phosphatase; LDH: lactate dehydrogenase.

institutional review board at each institution. A waiver of informed consent was granted by the institutional review boards on the condition that all patients were provided with the right to opt-out of the study. The eligibility criteria included: (i) histopathologically diagnosed carcinoma of the prostate; (ii) confirmed failure of primary ADT; and (iii) age $\geq 20$ years.

Treatment. Cabazitaxel was administered according to a 3- or 4weekly $\left(20-25 \mathrm{mg} / \mathrm{m}^{2}\right)$ regimen based on the schedules reported in the TROPIC (6) and PROSELICA (7) trials, and only one case was treated with $15 \mathrm{mg} / \mathrm{m}^{2}$ cabazitaxel. Prednisolone $(5 \mathrm{mg}$ ) was administered twice daily simultaneously with medical or surgical castration. The dose and schedule of cabazitaxel were modified according to the severity of any adverse events (AEs) in each case. Treatment with cabazitaxel was continued according to physician judgement, based on disease progression, AEs, or patient refusal.

Endpoints. Progressive disease was defined as an increase in serum prostate-specific antigen (PSA) of $>2 \mathrm{ng} / \mathrm{ml}$ and a $50 \%$ increase over the nadir, and/or the appearance of a new lesion or progression of one or more known lesions classified by the Response Evaluation Criteria in Solid Tumours Version 1.1 (8). Radiographic progression was defined as progression of measurable disease or bone scan progression. Clinically significant pain was defined by daily consumption of narcotic or non-narcotic analgesics for pain derived from prostate cancer. Performance status was determined by the Eastern Cooperative Oncology Group criteria.
Statistical analysis. All statistical analyses were performed using JMP version 13 software (SAS Institute, Cary, NC, USA). The Kruskal-Wallis test and Wilcoxon exact test were used to analyse PSA response and survival between groups. Progression-free survival and overall survival were determined by the Kaplan-Meier method and the log-rank test was used to compare survival duration between groups. All tests were two-sided, and values of $p<0.05$ were considered to indicate statistically significant differences.

\section{Results}

Seventy-four patients treated with cabazitaxel chemotherapy were identified. Among them, 62 were treated with ARAT agents before cabazitaxel chemotherapy and 12 were not. The patient characteristics are presented in Table I. Similar clinicopathological characteristics were observed in the patients with and without prior use of ARAT agents.

PSA response, defined as $>50 \%$ PSA decline, was recognised in $1(9.1 \%)$ and $15(26.8 \%)$ patients without and with prior use of ARAT agents, respectively. The PSA declines were comparable among patients with and without prior use of ARAT agents ( $p=0.33$; Figure 1A). Progressionfree survival was comparable between patients with and without prior ARAT agents ( $p=0.98$; Figure 1B). Overall survival was also comparable between patients with and without prior ARAT agents ( $p=0.42$; Figure 1C). 
A

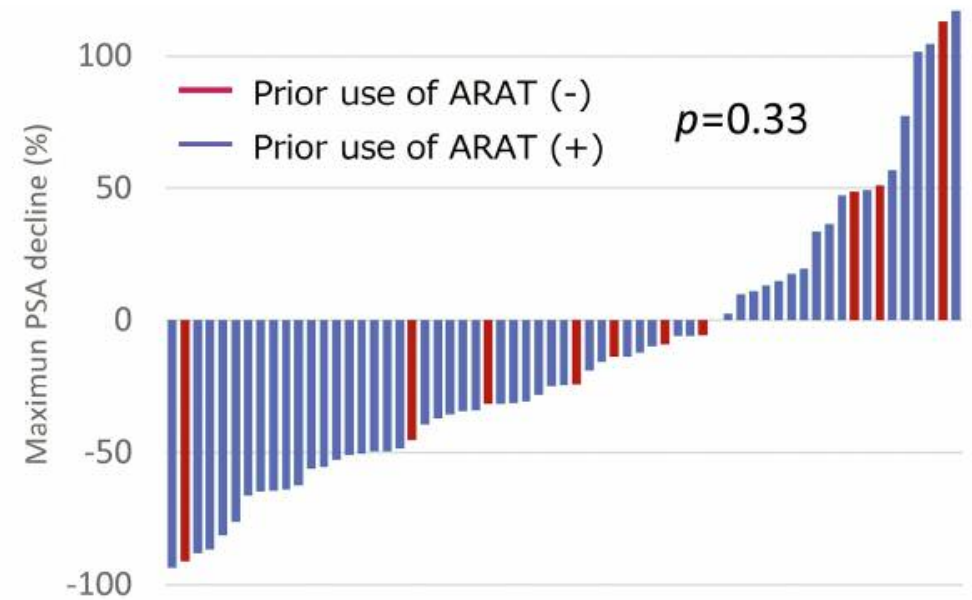

B

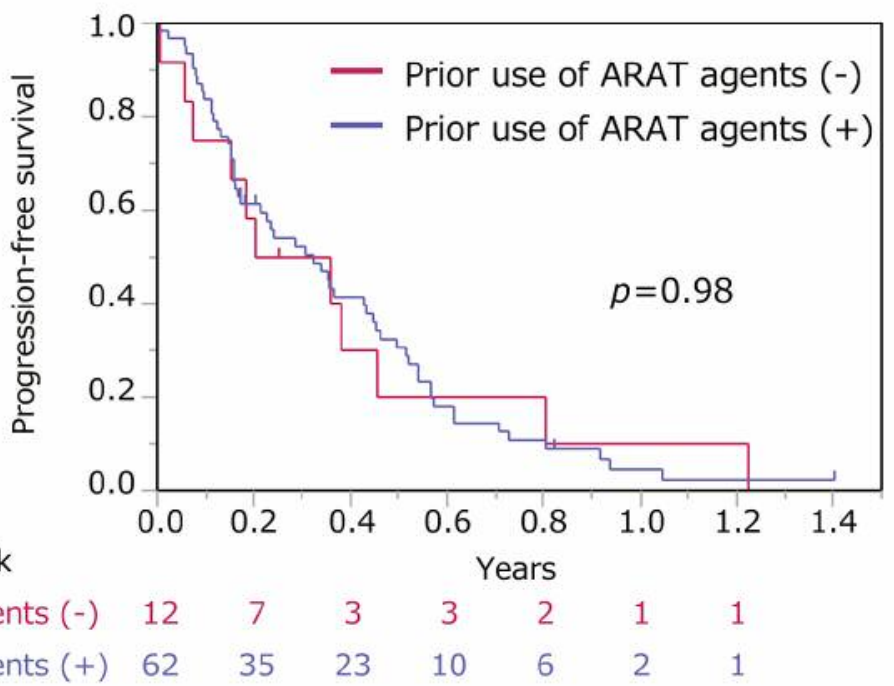

C

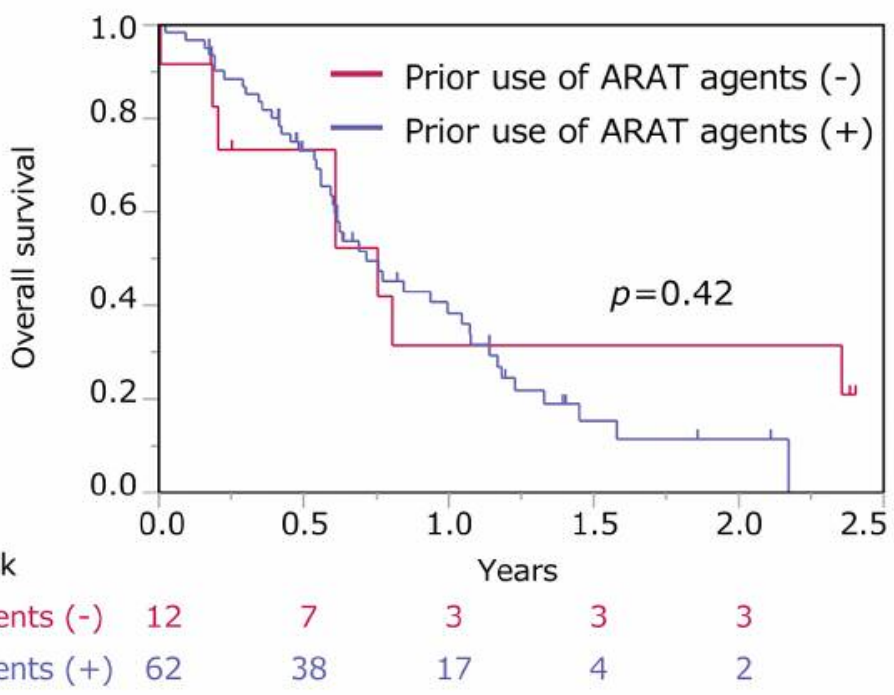

Figure 1. Clinical outcomes of cabazitaxel chemotherapy in patients with or without prior use of ARAT agents. (A-C) Best PSA response (A), progression-free survival $(B)$, and overall survival $(C)$ in patients with metastatic CRPC treated with cabazitaxel chemotherapy according to prior use of ARAT agents. 


\section{Discussion}

Prior treatment with ARAT agents has been reported to be associated with worse outcome following docetaxel chemotherapy $(2,3)$. Preclinical studies on prostate cancer cells demonstrated cross-resistance between taxanes and ARAT agents. Furthermore, van Soest et al. have revealed collateral resistance to taxanes such as docetaxel and cabazitaxel in enzalutamide- and abiraterone-resistant PC346 cells (4). Similarly, we have recently reported that enzalutamide-resistant $22 \mathrm{Rv} 1$ cells were resistant to taxanes including docetaxel and cabazitaxel (3). Thus, collateral resistance to docetaxel after ARAT agents can be supported by findings from in vitro preclinical studies.

Meanwhile, van Soest et al. have reported, in an in vivo study, that enzalutamide-resistant PC346C cells showed collateral resistance to docetaxel, but surprisingly not to cabazitaxel (4). Similarly, Al Nakouzi et al. have observed no collateral resistance to cabazitaxel in LNCaP-derived enzalutamide-resistant cells established by an in vivo system (9). As a possible mechanism for the lack of collateral resistance to cabazitaxel after ARAT agents, higher accumulation of cabazitaxel in tumour tissues in vivo compared with docetaxel has been suggested (10).

Consistent with the preclinical studies in vivo, cabazitaxel was suggested to be active after ARAT agents in the postdocetaxel setting. Al Nakouzi et al. and van Soest et al. have observed comparable antitumor effects and prognoses among patients with and without prior ARAT agents in cabazitaxel chemotherapy $(9,11)$. Similarly, the present study showed comparable PSA responses and prognoses among patients with and without prior ARAT agents, supporting the activity of cabazitaxel chemotherapy even after prior use of ARAT agents and docetaxel. Notably, cabazitaxel showed survival benefits in the era before ARAT agents emerged. Therefore, the survival benefits of cabazitaxel in the era of ARAT agents have not been proven. However, based on these real-world observations, cabazitaxel is expected to prolong survival even in the era of ARAT agents.

Thus, cabazitaxel is suggested to be useful by retaining antitumor efficacy after prior use of ARAT agents compared with docetaxel. However, the following points require attention: (i) cabazitaxel is usually utilised after docetaxel; (ii) the standard dose of cabazitaxel $\left(25 \mathrm{mg} / \mathrm{m}^{2}\right)$ differs from that of docetaxel $\left(75 \mathrm{mg} / \mathrm{m}^{2}\right)$; and (iii) cabazitaxel was approved after ARAT agents became clinically available in Japan, while docetaxel had already been approved before clinical use of ARAT agents. In addition, the present study had some limitations including its retrospective design, limited number of patients, and nature of multi-institution study.

\section{Conclusion}

Taken together, the present study demonstrated no prognostic impact of prior ARAT agents in cabazitaxel chemotherapy after docetaxel, suggesting that cabazitaxel can be expected to remain active even after prior use of ARAT agents and docetaxel.

\section{Conflicts of Interest}

The Authors declare no conflicts of interest regarding this study.

\section{Authors' Contributions}

MS designed the study, analyzed the data, and wrote the draft of the manuscript. All other Authors have contributed to data collection and interpretation, and critically reviewed the manuscript. EM supervised the study.

\section{Acknowledgements}

This work was supported by a JSPS KAKENHI grant (17K11145). The Authors would like to acknowledge the contributions of the following collaborators: Akito Yamaguchi at Harasanshin Hospital (Fukuoka), Takashi Dejima at Kyushu Central Hospital (Fukuoka), Satoshi Otsubo at Kitakyushu Municipal Medical Center (Kitakyushu), Akio Tsutsui at JCHO Kyushu Hospital (Kitakyushu), and Yoshifumi Hori at Miyazaki Prefectural Miyazaki Hospital (Miyazaki). The Authors also thank Alison Sherwin, PhD, from Edanz Group (www.edanzediting.com/ac) for editing a draft of this manuscript.

\section{References}

1 Damodaran S, Lang JM and Jarrard DF: Targeting metastatic hormone sensitive prostate cancer: Chemohormonal therapy and new combinatorial approaches. J Urol 201: 876-885, 2019. PMID: 30747897. DOI: 10.1097/JU.0000000000000117

2 Schweizer MT, Zhou XC, Wang H, Bassi S, Carducci MA, Eisenberger MA and Antonarakis ES: The influence of prior abiraterone treatment on the clinical activity of docetaxel in men with metastatic castration-resistant prostate cancer. Eur Urol 66: 646-652, 2014. PMID: 24491307. DOI: 10.1016/j.eururo. 2014.01.018

3 Shiota M, Dejima T, Yamamoto Y, Takeuchi A, Imada K, Kashiwagi E, Inokuchi J, Tatsugami K, Kajioka S, Uchiumi T and Eto M: Collateral resistance to taxanes in enzalutamideresistant prostate cancer through aberrant androgen receptor and its variants. Cancer Sci 109: 3224-3234, 2018. PMID: 30051622. DOI: $10.1111 /$ cas.13751

4 van Soest RJ, de Morrée ES, Kweldam CF, de Ridder CMA, Wiemer EAC, Mathijssen RHJ, de Wit R and van Weerden WM: Targeting the androgen receptor confers in vivo cross-resistance between enzalutamide and docetaxel, but not cabazitaxel, in castration-resistant prostate cancer. Eur Urol 67: 981-985, 2015. PMID: 25484141. DOI: 10.1016/j.eururo.2014.11.033

5 van Soest RJ, van Royen ME, de Morrée ES, Moll JM, Teubel W, Wiemer EA, Mathijssen RH, de Wit R and van Weerden WM: Cross-resistance between taxanes and new hormonal 
agents abiraterone and enzalutamide may affect drug sequence choices in metastatic castration-resistant prostate cancer. Eur J Cancer 49: 3821-3830, 2013. PMID: 24200698. DOI: 10.1016 /j.ejca.2013.09.026

6 de Bono JS, Oudard S, Ozguroglu M, Hansen S, Machiels JP, Kocak I, Gravis G, Bodrogi I, Mackenzie MJ, Shen L, Roessner M, Gupta S and Sartor AO; TROPIC Investigators: Prednisone plus cabazitaxel or mitoxantrone for metastatic castrationresistant prostate cancer progressing after docetaxel treatment: a randomised open-label trial. Lancet 376: 1147-1154, 2010. PMID: 20888992. DOI: 10.1016/S0140-6736(10)61389-X

7 Eisenberger M, Hardy-Bessard AC, Kim CS, Géczi L, Ford D, Mourey L, Carles J, Parente P, Font A, Kacso G, Chadjaa M, Zhang W, Bernard J and de Bono J: Phase III study comparing a reduced dose of cabazitaxel $\left(20 \mathrm{mg} / \mathrm{m}^{2}\right)$ and the currently approved dose $\left(25 \mathrm{mg} / \mathrm{m}^{2}\right)$ in postdocetaxel patients with metastatic castration-resistant prostate cancer-PROSELICA. J Clin Oncol 35: 3198-3206, 2017. PMID: 28809610. DOI: 10.1200/JCO.2016.72.1076

8 Scher HI, Halabi S, Tannock I, Morris M, Sternberg CN, Carducci MA, Eisenberger MA, Higano C, Bubley GJ, Dreicer R, Petrylak D, Kantoff P, Basch E, Kelly WK, Figg WD, Small EJ, Beer TM, Wilding G, Martin A and Hussain M; Prostate Cancer Clinical Trials Working Group: Design and end points of clinical trials for patients with progressive prostate cancer and castrate levels of testosterone: recommendations of the Prostate Cancer Clinical Trials Working Group. J Clin Oncol 26: 11481159, 2008. PMID: 18309951. DOI: 10.1200/JCO.2007.12.4487
9 Al Nakouzi N, Le Moulec S, Albigès L, Wang C, Beuzeboc P, Gross-Goupil M, de La Motte Rouge T, Guillot A, Gajda D, Massard C, Gleave M, Fizazi K and Loriot Y: Cabazitaxel remains active in patients progressing after docetaxel followed by novel androgen receptor pathway targeted therapies. Eur Urol 68: 228235, 2015. PMID: 25012524. DOI: 10.1016/j.eururo.2014.04.015

10 de Morrée ES, Böttcher R, van Soest RJ, Aghai A, de Ridder CM, Gibson AA, Mathijssen RH, Burger H, Wiemer EA, Sparreboom A, de Wit R and van Weerden WM: Loss of SLCO1B3 drives taxane resistance in prostate cancer. Br J Cancer 115: 674-681, 2016. PMID: 27537383. DOI: 10.1038/bjc.2016.251

11 van Soest RJ, Nieuweboer AJ, de Morrée ES, Chitu D, Bergman AM, Goey SH, Bos MM, van der Meer N, Hamberg P, de Wit $\mathrm{R}$ and Mathijssen RH; Dutch Uro-Oncology Studygroup (DUOS): The influence of prior novel androgen receptor targeted therapy on the efficacy of cabazitaxel in men with metastatic castration-resistant prostate cancer. Eur J Cancer 51: 2562-2569, 2015. PMID: 26278646. DOI: 10.1016/j.ejca.2015.07.037

Received November 18, 2019

Revised November 22, 2019

Accepted November 25, 2019 